\title{
optica
}

\section{Conservation of photon rate in endothermic photoluminescence and its transition to thermal emission: supplementary material}

\author{
A. MAnOR, ${ }^{1}$ LeOpoldo MARTIn, ${ }^{2}$ And CARMel RotSChILD ${ }^{1,2 *}$ \\ ${ }^{1}$ Russell Berrie Nanotechnology Institute, Technion - Israel Institute of Technology, Haifa 32000, Israel \\ ${ }^{2}$ Department of Mechanical Engineering, Technion - Israel Institute of Technology, Haifa 32000, Israel \\ *Corresponding author: carmelr@.technion.ac.il \\ Published 19 June 2015
}

This document provides supplementary information to "Conservation of photon rate in endothermic photoluminescence and its transition to thermal emission," http://dx.doi.org/10.1364/optica.2.000585. It is divided to two sections, giving specific data on the issues of fluorescence intensity ratio thermometry (FIR) and the chemical potential measurement method. (C) 2015 Optical Society of America

http://dx.doi.org/10.1364/optica.2.000585.s001

The Fluorescence Intensity Ratio Thermometry (FIR) method:

Using the FIR method, temperature can be measured by recording the intensity ratio of two adjacent optical emission peaks of a luminescent sample. Under the condition of full thermalization of electrons to the band edge, one can apply the Boltzmann population statistics, to the peaks ratio:

(a)

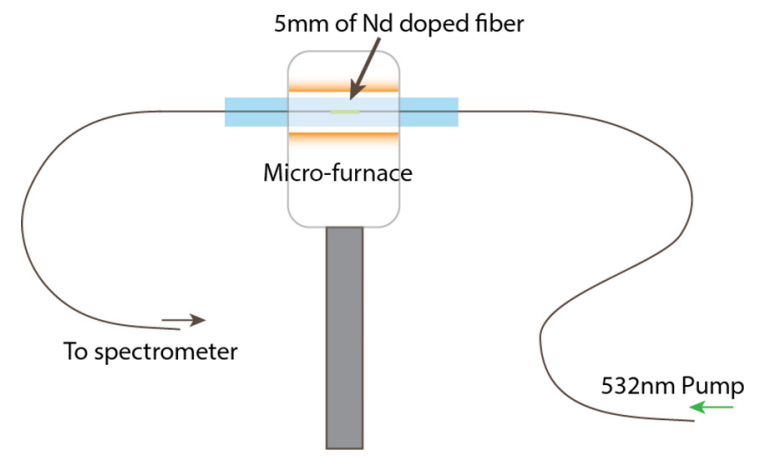

$$
R=C \cdot e^{\left(-\frac{\Delta E}{K_{b} T}\right)}
$$

where $\Delta E$ is the energy difference between the two emission peaks. The constant $C$ is determined by the density of states and the matrix element for optical transitions between the states. Once $\mathrm{C}$ is known experimentally, the relation can be used to determine the sample's temperature during the experiment. In order to find $\mathrm{C}$, we place the sample in a calibrated micro-furnace, which

(b)

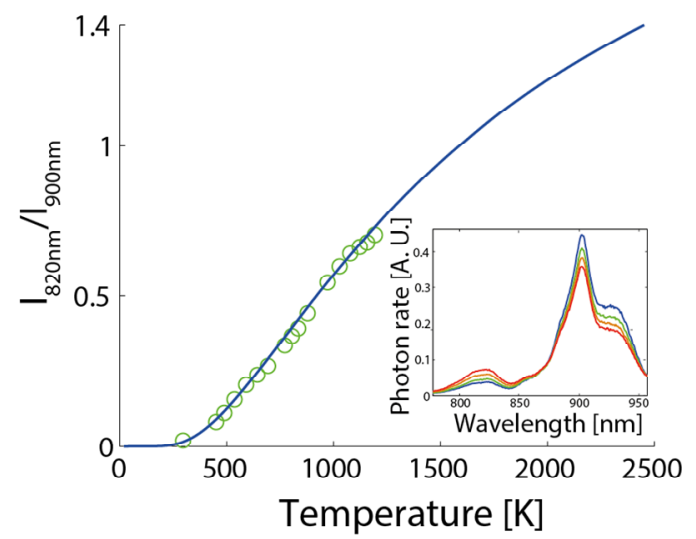

Fig. S1. (a) The FIR measurement experimental setup (b) and the intensity ratio vs. temperature plot. 
consists of a heated Quartz cylinder. From the same Neodymium $\left(\mathrm{Nd}^{+3}\right)$ doped Silica fiber as in the experiment, we cut a $5 \mathrm{~mm}$ long fiber and connect (fuse) it, from both sides, to a long passive optical fiber in order to guide its emission (Figure S1a). The $5 \mathrm{~mm}$ active fiber section is placed at the center of the furnace, while the fiber is pumped by 532nm laser from one side, and light is collected from its other side. A spectrum analyzer is used to measure the ratio between the $900 \mathrm{~nm}$ and $820 \mathrm{~nm}$ peaks as function of the furnace temperature. Figure S1b depicts the experimental intensity-ratio to temperature plot obtained from the measurement, and the fitted curve. The constant is found to be $\mathrm{C}=2.6$. The inset shows the evolution of the peak ratio shift with temperature increase.

\section{Calculation of the chemical potential from the experimental data:}

The chemical potential can be calculated from equation (S1):

$$
\mu(\hbar \omega, T)=K_{b} T \ln \left(\frac{R(\hbar \omega, T)}{\varepsilon(\hbar \omega) R_{0}(\hbar \omega, T)}\right)
$$

Since $\mu$ is constant at the relevant emission band between $600 \mathrm{~nm}$ $1000 \mathrm{~nm}$, it can be extracted by integrating the experimental emitted photon rate in the band under PL and thermal excitation presented in figure 2c (blue and red lines):

$$
\mu(\hbar \omega, T)=K_{b} T \ln \left(\frac{\int_{\text {band }} R_{p l}(\hbar \omega, T) d \hbar \omega}{\int_{\text {band }} R_{\text {thermal }}(\hbar \omega, T) d \hbar \omega}\right)
$$

(S3)

For temperatures below $1150 \mathrm{~K}$, where the thermal emission signal is below our detection limit, the experimental data for the thermal emission was extrapolated to $300 \mathrm{~K}$ as follows. The sample's relative emissivity was calculated as the ratio between the thermal emission in the experiment and the theoretical black body emission rate at the same temperatures:

$$
\varepsilon(\hbar \omega)=\frac{R_{\text {thermal }}(\hbar \omega, T)}{R_{0}(\hbar \omega, T)}
$$

The result is shown in figure $\mathrm{S} 2$.

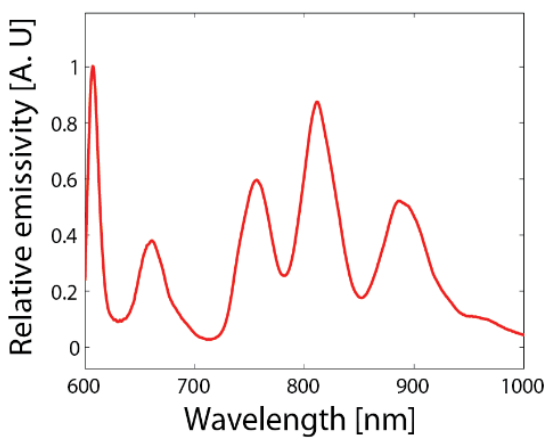

Fig. S2. Measured relative emissivity of $\mathrm{Nd}: \mathrm{SiO} 2$ sample.
The thermal photon rate for the temperature range $300 \mathrm{~K}-1150 \mathrm{~K}$ is then calculated by:

$$
R_{\text {thermal_extrapolated }}(\hbar \omega, T)=\int_{\text {band }} \varepsilon(\hbar \omega) \cdot R_{0}(\hbar \omega, T) d \hbar \omega
$$

